\title{
The role of the third acid-fast bacillus smear in tuberculosis screening for infection control purposes: A controversial topic revisited
}

\author{
A Wilmer $M D^{1,2}$, E Bryce $M D^{1,2}$, J Grant $M \operatorname{MCM}^{1,2}$
}

\begin{abstract}
A Wilmer, E Bryce, J Grant. The role of the third acid-fast bacillus smear in tuberculosis screening for infection control purposes: A controversial topic revisited. Can J Infect Dis Med Microbiol 2011;22(1):e1-e3.
\end{abstract}

INTRODUCTION: Recent studies have suggested that two negative acid-fast bacillus (AFB) smears may be as effective as three when screening patients with suspected Mycobacterium tuberculosis for respiratory isolation purposes. However, current recommendations in Canada, the United States and Europe still support a three-smear approach.

METHODS: The microbiology database of a tertiary care hospital was searched for sputum, tracheal aspirates and bronchoalveolar lavage samples from 2003 to 2007 that had been sent for mycobacterial testing. The first patient specimen to become AFB smear positive was noted. As well, the time required to collect the third specimen in hospitalized patients who remained smear negative was used to estimate the savings in isolation costs associated with a two-smear approach.

RESULTS: There were 8347 respiratory specimens from 5168 patients in the five-year period. Of these patients, 2.2\% (116 of 5168) were AFB smear positive, of whom 55.2\% (64 of 116) were culture positive for Mycobacterium tuberculosis. Overall 89\% (57 of 64) of patients were identified as being AFB smear positive by the first smear, $7.8 \%$ (five of 64 ) were identified by the second smear and 3.2\% (two of 64) were identified by further smears. Smear-negative patients spent a combined 710 days in isolation awaiting collection of the third sample at a cost of approximately $\$ 142,000$ over five years.

CONCLUSION: A two-smear approach for discontinuation of respiratory isolation precautions is safe and has the potential to reduce hospital expenditures.

Key Words: Humans; Infection control; Retrospective studies; Sputum/ microbiology; Tuberculosis/microbiology; Tuberculosis/prevention and control
Le rôle du troisième frottis de bacille acidorésistant dans le dépistage de la tuberculose pour le contrôle de l'infection : Un sujet controversé revisité

INTRODUCTION : De récentes études laissent supposer que deux frottis négatifs au bacille acidorésistant (BAR) peuvent être aussi efficaces que trois frottis pour isoler les bacilles respiratoires des patients atteints d'un Mycobacterium tuberculosis présumé. Cependant, au Canada, aux ÉtatsUnis et en Europe, on recommande d'effectuer trois frottis.

MÉTHODOLOGIE : Les auteurs ont effectué une recherche dans la base de données microbiologiques d'un hôpital de soins tertiaires pour retracer les échantillons d'expectorations, d'aspirations trachéales et de lavages bronchoalvéolaires prélevés entre 2003 et 2007 qui avaient fait l'objet d'un test mycobactérien. Le premier échantillon prélevé sur les patients dont le frottis devenait positif au BAR était pris en note. De plus, le délai nécessaire pour prélever le troisième frottis chez les patients hospitalisés dont le frottis demeurait négatif a permis d'évaluer les économies sur les coûts d'isolement associés à une démarche à deux frottis.

RÉSULTATS : Pendant la période de cinq ans, 8347 échantillons respiratoires ont été prélevés auprès de 5168 patients. De ce nombre, 2,2 \% (116 sur 5 168) étaient positifs au frottis de BAR, et la culture de 55,2\% (64 sur 116) d'entre eux était positive au Mycobacterium tuberculosis. Dans l'ensemble, le frottis de BAR de $89 \%$ (57 sur 64) des patients a été déterminé comme positif au premier frottis, celui de 7,8 \% (cinq sur 64), au deuxième frottis, et celui de 3,2 \% (deux sur 64) au moins au troisième frottis. Les patients dont le frottis était négatif ont passé une période de 710 jours combinés en isolement dans l'attente du prélèvement du troisième échantillon, au coût d'environ 142000 \$ répartis sur cinq ans. CONCLUSION : L'exécution de deux frottis pour décider de mettre un terme aux précautions respiratoires est sécuritaire et pourrait réduire les dépenses des hôpitaux.
$\mathrm{T}$ raditionally, three negative acid-fast bacillus (AFB) sputum smears are recommended before a patient with suspected pulmonary Mycobacterium tuberculosis (MTB) can be removed from isolation. Recent research $(1,2)$ demonstrated that the first two AFB sputum smears identify the majority of smear-positive patients, suggesting that the third smear is usually not helpful in deciding when to discontinue isolation precautions. Despite this evidence, a three-smear approach for isolation precautions is still recommended in Canada, the United States and Europe (3-5)
A retrospective study of all sputum samples, tracheal aspirates and bronchoalveolar lavage (BAL) specimens collected between January 1, 2003 and December 31, 2007, was performed to determine whether patients who were AFB smear negative after two smears were likely to remain negative on the third smear. The use of the third AFB smear for determination of the need for isolation was also assessed. The costs associated with performing the third AFB smear and with maintaining respiratory isolation while awaiting results, were also calculated.

${ }^{1}$ Department of Medicine, University of British Columbia; ${ }^{2}$ Department of Microbiology, Vancouver General Hospital, Vancouver, British Columbia

Correspondence: Dr A Wilmer, clo Department of Microbiology, JPPN 1110, Vancouver Hospital and Health Sciences Centre, 855 West

12th Avenue, Vancouver, British Columbia V5Z 1M9. Telephone 778-228-5395, fax 604-875-4359, e-mail avwilmer@gmail.com 


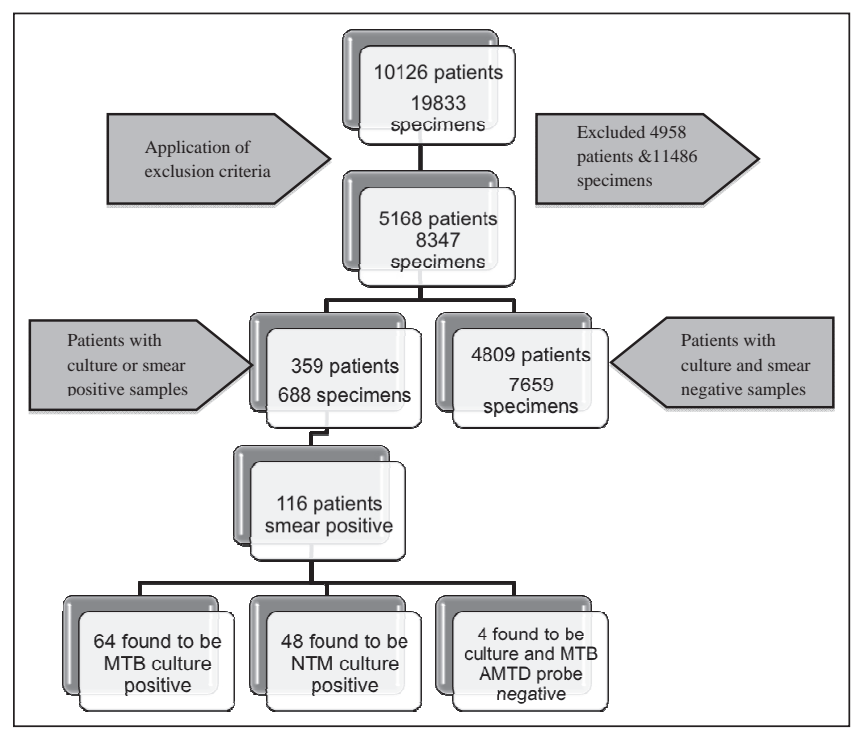

Figure 1) Summary of the extracted data. MTB Mycobacterium tuberculosis; NTM Nontuberculosis mycobacteria

\section{METHODS}

Study site

The present study was conducted in the medical microbiology laboratory of a university tertiary care hospital in Vancouver, British Columbia, where the MTB rate is 14 cases $/ 100,000$ population. The laboratory serves the study hospital and four secondary acute care hospitals (2410 beds), as well as four emergency departments and four hospital outpatient laboratories, processing approximately 4000 mycobacterial samples annually. The study institution identifies 30 new cases of MTB annually, corresponding to a high-risk facility, by established guidelines (3). Within the study hospital, inpatients spend a total of more than 300 days/year in respiratory isolation for suspected MTB, excluding patients admitted directly to the specialized MTB ward.

\section{Specimen processing}

Up to $10 \mathrm{~mL}$ of sputum specimens were collected and processed using a NALC-NaOH digestion/decontamination method (6). Specimens were stained with Auramine $\mathrm{O}$ for fluorescent microscopy, decontaminated and cultured using standard laboratory methods on solid media and with the BACTEC MGIT 960 Detection System (Becton Dickinson Microbiology Systems, USA) (6). Smear-positive specimens were tested with the AMTD probe (Gen-Probe Incorporated, USA). Culture-positive samples were confirmed as MTB by TB Accuprobe (Gen-Probe Incorporated, USA) and Niacin nitrate testing. Heat shock protein polymerase chain reaction followed by sequencing and gene BLAST analysis identified nontuberculosis mycobacteria. All tests on culture-positive samples were performed at a reference laboratory.

\section{Data extraction}

Data from January 1, 2003 to December 31, 2007, were extracted from the laboratory database. Sputum, tracheal aspirates and BAL specimens were retained, while nonrespiratory and biopsy specimens were removed from the data set. Any samples rejected by the laboratory for technical or quality reasons were also excluded, as were routine pretransplant samples from lung transplant donors. All pretransplant samples were smear and culture negative.

For patients in the dataset who repeatedly tested smear or culture positive for mycobacterial species, only the specimens collected within the initial two-week period of the patient becoming culture or smear positive were considered. For patients in the dataset who were repeatedly found to be AFB smear and culture negative, only the specimens collected within the two-week period of the initial specimen collection date were included. The number of days between collection of the second and third specimens from inpatients at the study hospital was calculated to determine the additional days that the patients remained on airborne isolation precautions while awaiting results of the third AFB smear.

\section{Cost calculations}

The additional charge associated with a private room at the study hospital was used to estimate the cost of isolating a patient (\$200). The provincial billing allowance for an AFB smear ( $\$ 7.25$ per smear) was used to determine laboratory costs associated with the third AFB smear.

\section{Isolation policy at the study hospital}

The isolation policy at the study hospital adheres to the Canadian Tuberculosis standards (3). Patients who have had specimens collected to rule out MTB are automatically placed on airborne isolation precautions and remain in a negative pressure room until the third specimen tests negative for AFB by microscopy. One of these specimens should be collected in the morning and, ideally, all should be collected at least $8 \mathrm{~h}$ apart. If a patient is smear negative, but clinical suspicion merits further investigation or empirical therapy for MTB, isolation is continued until there is an alternative diagnosis or until two weeks of therapy with clinical improvement has occurred.

\section{RESULTS}

The initial query identified 19,833 specimens sent for AFB analysis. After removal of nonrespiratory specimens from the initial database query, 8347 samples from 5168 patients remained (Figure 1).

A single specimen was collected from 3674 patients, while 455 patients had two specimens, 623 had three and 416 had four or more collected. Of these patients, $2.2 \%$ (116 of 5168) were AFB smear positive, of which $55.2 \%$ (64 of 116 ) were culture positive for MTB; $41.4 \%$ (48 of 116) were culture positive for nontuberculosis mycobacteria. There were four smear-positive patients who were culture negative and had a negative AMTD probe for MTB.

Of the AFB smear-positive patients who were culture positive for MTB, $89 \%$ (57 of 64) were identified with the first smear, $7.8 \%$ (five of 64) were identified on the second smear and a further 3.2\% (two of 64) were identified on at least the third smear.

A review of smear-negative inpatients revealed that 39.9\% (299 of 750 ) had the second and third specimens collected on the same day; $44.8 \%$ (336 of 750 ) had a one-day interval, $8.7 \%$ (65 of 750 ) had a two-day interval and 6.7\% (115 of 750) had at least a three-day interval between collection of the second and third specimens. Assuming that all samples were processed on the day of collection, a combined 710 inpatient days were spent in isolation waiting for the collection of the third specimen. The average delay in waiting for collection of the third specimen was 0.95 days/patient ( 710 days/750 inpatients). This led to an estimated cost of $\$ 142,000$ for isolation over five years, plus an estimated $\$ 5,437.50$ in laboratory costs for processing the third AFB smears.

\section{DISCUSSION}

With escalating health care costs, facilities should examine every avenue to ensure that a patient's disposition is efficient and that resources are allocated wisely. The present study investigated the potential impact of a two-smear airborne isolation policy in a tertiary care hospital, in terms of cost savings and possible risk of missing a smear-positive patient.

Four comparable studies $(1,2,7,8)$ that used similar methods have been performed. The percentages of patients in these studies, identified as AFB smear positive by at least the third specimen alone, were $0 \%$, $5.3 \%, 8.6 \%$ and $11.1 \%(1,2,7,8)$. Two of these studies endorsed change to a two-smear approach for discontinuing isolation based on their 
findings, while the other two recommended continuing a three-smear approach $(1,2,7,8)$. The present study identified $3.2 \%$ of patients on at least the third smear, which supports a two-smear approach as sufficient for discontinuing respiratory isolation.

Isolating patients is costly, and negatively impacts their hospital stay (9). The ideal collection of three samples for AFB smear stipulates that they should be collected $8 \mathrm{~h}$ apart (3). This rarely occurs; therefore, the 0.95 day average between collection of the second and the third sample is a reflection of actual practice. Discontinuing isolation after the second negative smear would have resulted in 142 fewer isolation days and saved an estimated $\$ 28,400$ annually, while still detecting $96.8 \%$ (62 of 64) of all MTB smear-positive patients. This result is similar to the study by Craft et al (1) who estimated an annual saving of $\$ 32,000$ in isolation costs using a two-smear approach. The additional laboratory savings from a two-smear approach would be $\$ 1,087.50$ annually. It should be noted that these costs likely underestimate the potential savings because they do not include the costs of personal protective equipment, operation of negative pressure rooms and nursing time.

Applying a two-smear approach for respiratory isolation would have led to two missed cases of AFB smear-positive MTB over the five-year period of the present study. A three-smear approach would have still missed one of these two cases. When the specimens from these two patients were reviewed, both became smear positive on BAL after being smear negative on several proceeding sputa. Given that AFB specimens could only be isolated using invasive collection techniques, it could be hypothesized that they had a lower bacillary burden and, may in fact, have carried the same risk of transmission as smear-negative patients. Regardless, these patients would have remained on respiratory isolation as per the study hospital's policy if the clinical suspicion of MTB remained high. Because of the serious ramifications of an MTBpositive patient being removed from isolation after two negative AFB smears, it is important to emphasize that isolation policies should always consider the patient's clinical status and risk of MTB transmission in combination with laboratory findings.

An important difference between the present study and others was the inclusion of BAL specimens and tracheal aspirates, in addition to sputum samples, because these are all integral to the clinical decisionmaking process regarding isolation and treatment. Four comparable studies $(1,2,7,8)$ only included sputum specimens when investigating this topic. Inclusion of these extra specimens makes the present study simpler to extrapolate to a clinical practice.

All patients, regardless of the total number of respiratory specimens collected, were included in the analysis. This includes screening and diagnostic samples. Three of the four comparable studies only included patients in their analysis if they had three or more smears performed, while the other did not specify how many smears were required for

\section{REFERENCES}

1. Craft DW, Jones MC, Blanchet CN, Hopfer RL. Value of examining three acid-fast bacillus sputum smears for removal of patients suspected of having tuberculosis from the "airborne precautions" category. J Clin Microbiol 2000;38:4285-7.

2. Mathew P, Kuo YH, Vazirani B, Eng RH, Weinstein MP. Are three sputum acid-fast bacillus smears necessary for discontinuing tuberculosis isolation? J Clin Microbiol 2002;40:3482-4.

3. Long R, Ellis E, eds. Canadian Tuberculosis Standards, 6th edn: Canadian Ministry of Health, 2007.

4. Taylor Z, Nolan CM, Blumberg HM; American Thoracic Society; Centers for Disease Control and Prevention; Infectious Diseases Society of America. Controlling tuberculosis in the United States. Recommendations from the American Thoracic Society, CDC, and the Infectious Diseases Society of America. MMWR Recomm Rep 2005;54(RR-12):1-81.

5. National Institute for Health and Clinical Excellence. Tuberculosis Clinical diagnosis and management of tuberculosis, inclusion $(1,2,7,8)$. This method could underestimate the value of a third specimen for patients in whom only one or two specimens were collected, by failing to detect patients who may have been positive if further samples were taken. However, once AFB are detected, most clinicians discontinue sampling. By eliminating patients with fewer than three smears performed, 64\% (41 of 64) of smear-positive MTB patients would have been eliminated because they were identified with the first or second smear. Excluding these patients from the present study would create a bias toward the importance of three-specimen screening. Because collection of three specimens for AFB smear does not always occur in the clinical setting, analysis of patients with any number of specimens collected is likely more applicable to clinical practice.

The present study has several limitations. First, similar to other comparable studies, chart review was not performed; consequently, no clinical data are provided. Because of this, we were not able to identify specific clinical factors associated with the patients who were not identified as being AFB smear positive until at least the third sputum sample. In addition, the hospital studied serves a very high-risk population including a $40 \%$ foreign-born population and a large disenfranchised inner-city population. The majority of HIV-infected patients are cared for at a different centre. Therefore, the pretest probability of tuberculosis may be higher than the average in Canada, but the rate of HIV may be lower than that of other major urban centres, making these numbers more difficult to extrapolate. However, the results of the present study remain consistent with many other studies; therefore, strengthening its findings.

To estimate the extra costs of respiratory isolation while awaiting the third AFB smear, the assumption was made that three negative AFB smears would have led to patient removal from respiratory isolation. In reality, this is a complex decision made by considering both clinical and laboratory findings; consequently, these patients may have been on respiratory isolation longer than estimated.

\section{CONCLUSIONS}

Considered as part of a risk-benefit calculation, a two-smear approach decreases time spent in respiratory isolation, benefiting the patient, direct care providers and infection control professionals, and leads to substantial cost savings, with minimal risk of tuberculosis transmission.

ACKNOWLEDGEMENTS: The authors thank James Chan for his assistance with data analysis and management.

CONFLICTS OF INTEREST: The authors have no conflicts of interest to declare.

and measures for its prevention and control. <http://www.nice.org. uk/nicemedia/pdf/CG033niceguideline.pdf> (Accessed on October 18, 2008).

6. Garcia LS, Isenberg HD, eds. Clinical Microbiology Procedures Handbook, 2nd edn update. Washington, DC: ASM Press, 2007.

7. Siddiqui AH, Perl TM, Conlon M, Donegan N, Roghmann MC. Preventing nosocomial transmission of pulmonary tuberculosis: When may isolation be discontinued for patients with suspected tuberculosis? Infect Control Hosp Epidemiol 2002;23:141-4.

8. Sprinson JE, Porco TC, Lawton ES, Mase SR, Flood JM. Yield of sputum smear examinations in evaluation of M. tuberculosis culture-positive pulmonary TB patients. Richmond: State of California Department of Health Services, Tuberculosis Control Branch 2006.

9. Stelfox HT, Bates DW, Redelmeier DA. Safety of patients isolated for infection control. JAMA 2003;290:1899-905. 


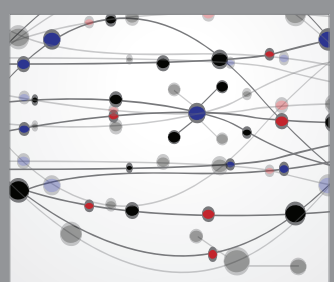

The Scientific World Journal
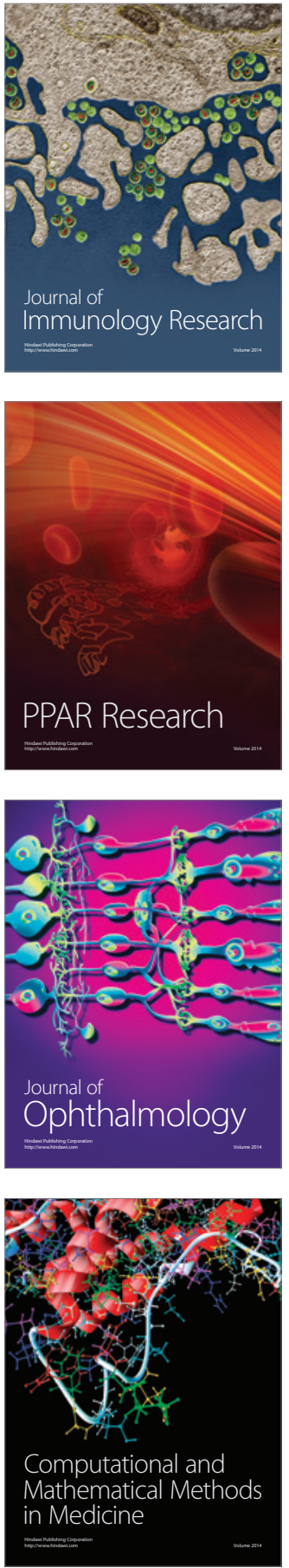

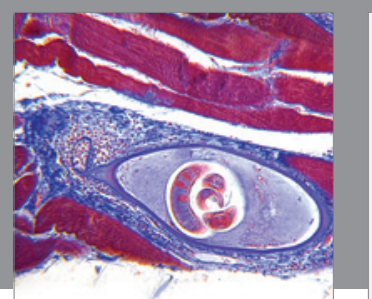

Gastroenterology Research and Practice

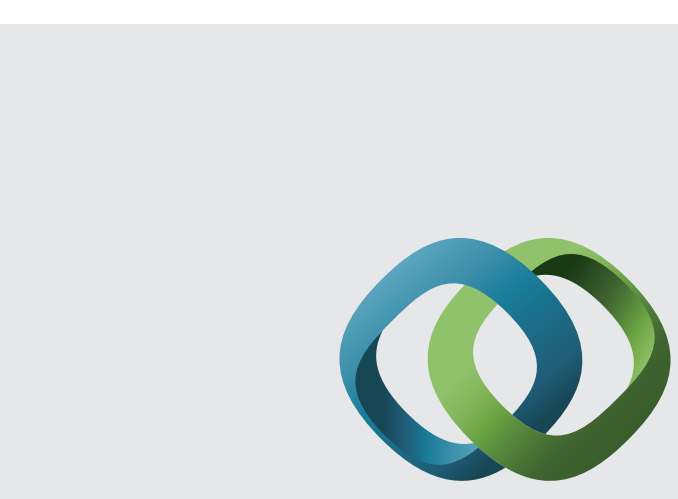

\section{Hindawi}

Submit your manuscripts at

http://www.hindawi.com
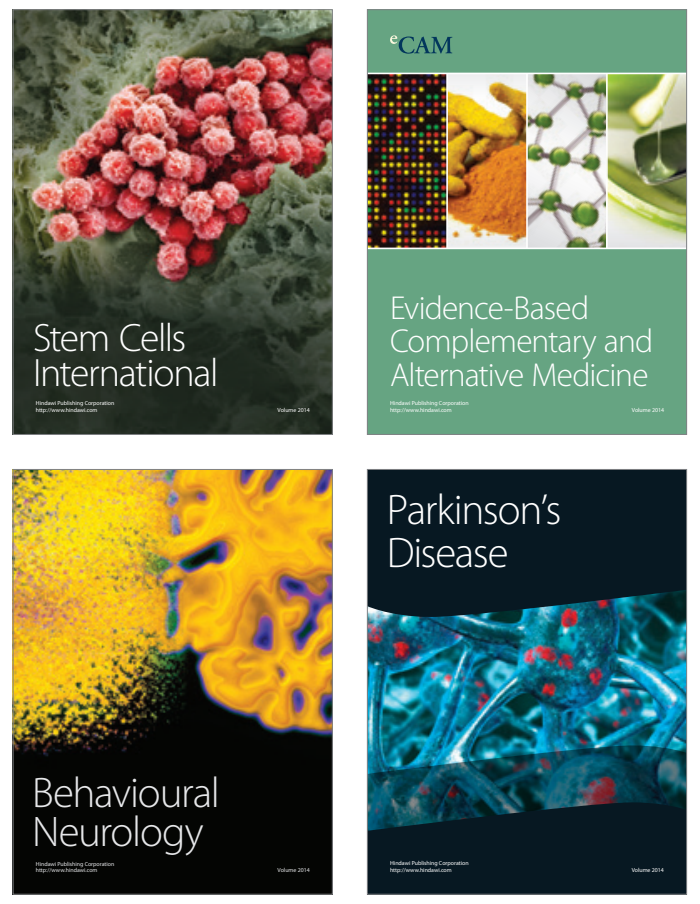
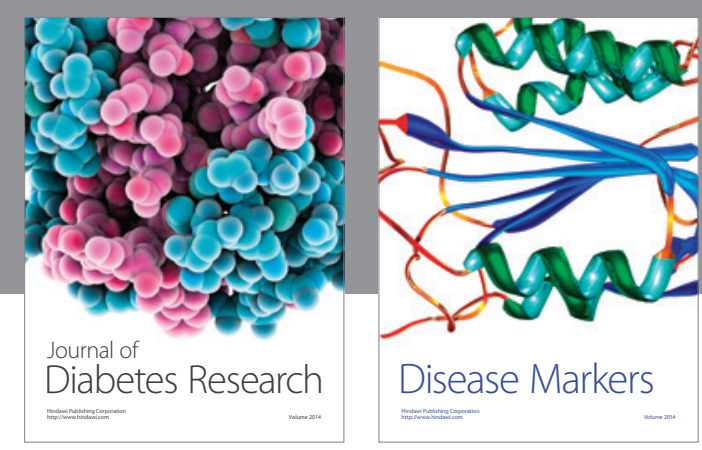

Disease Markers
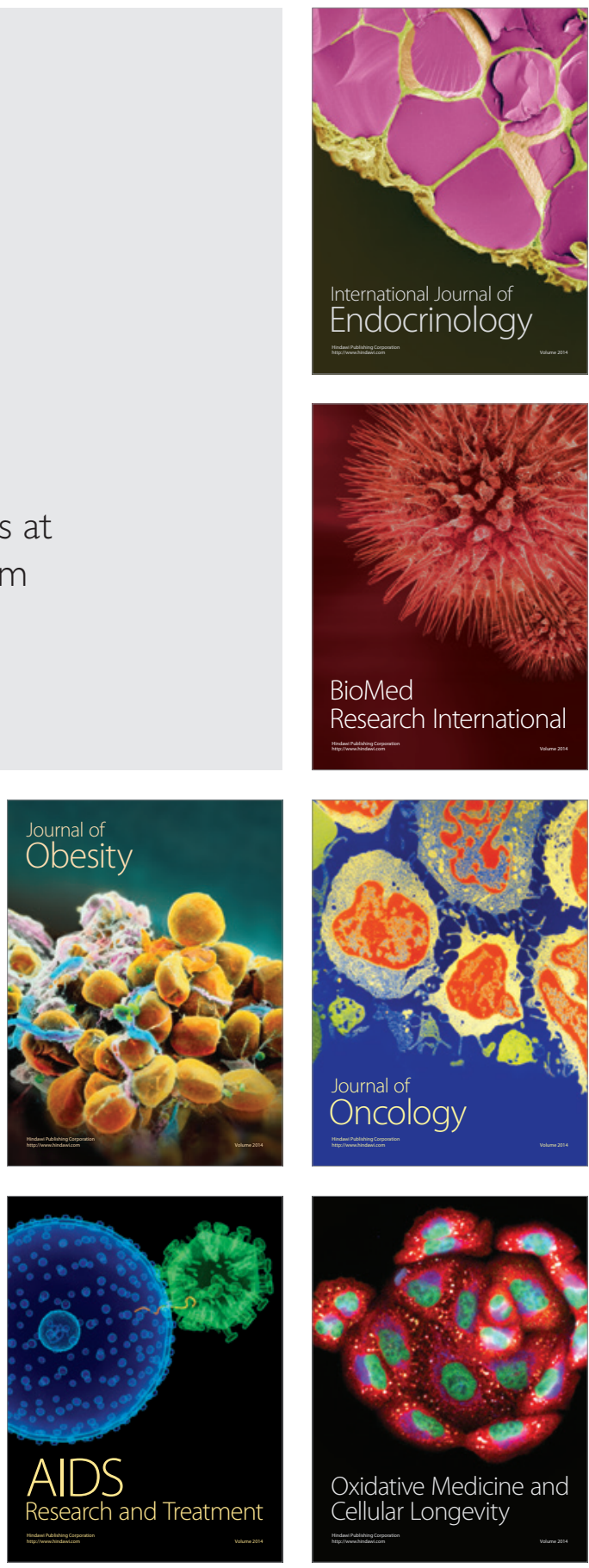